\title{
Towards a complete dispersive analysis of $\pi K$ scattering.
}

\author{
A. Rodas* \\ Departamento de Física Teórica and IPARCOS, Universidad Complutense de Madrid, 28040 \\ Madrid, Spain \\ E-mail: arodas@ucm.es
}

\section{J.R. Peláez}

Departamento de Física Teórica and IPARCOS, Universidad Complutense de Madrid, 28040 Madrid, Spain

E-mail: jrpelaezafis.ucm.es

In this talk we discuss the application of dispersion relations for low energy meson-meson scattering, in particular we review our ongoing dispersive analyses for the complete study of both $\pi K$ and $\pi \pi \rightarrow K \bar{K}$ channels, where some preliminary results for the former are shown.

The 9th International workshop on Chiral Dynamics

17-21 September 2018

Durham, NC, USA

${ }^{*}$ Speaker. 


\section{Introduction}

Pions and kaons are the lightest hadrons, corresponding to the pseudo-Goldstone bosons of the spontaneous chiral symmetry breaking sector of QCD. Thus by studying their interaction one is testing the QCD chiral symmetry breaking pattern. In addition, they appear in the final states in almost all hadronic interactions, which makes the understanding of their rescattering effects crucial. On top of that, most of the lightest mesons appear as resonances in this low energy meson-meson scattering channels. This is the case of the much debated $K_{0}^{*}(700)$ resonance, or the now well known $f_{0}(500)$, which are not considered to fit within the ordinary quark-antiquark picture.

Even though there are many different $\pi K$ scattering experiments $[1,2,3,4,5]$, none of them can directly access these interactions. All the main data sets are obtained by studying mesonnucleon to meson-meson-nucleon interactions. The desired pion-meson scattering is then obtained by assuming one pion exchange dominance, which could produce a large systematic effect depending on the accessible precision for the determination of the transferred momentum. As a result the literature is plagued with many conflicting data sets (see [6] for a recent review). Actually, this is the case for the most relevant partial waves in $\pi K$ scattering.

Apart from scattering itself, the description of the relevant resonances, in particular both the $f_{0}(500)$ and the $K_{0}^{*}(700)$ has been subject of debate for many years. These two particles are too broad to be determined with precision using simple models. In order to get a precise determination of their parameters, one needs to rely on suitable techniques for an analytic continuation far from the real axis, where the poles associated to such resonances are expected to be.

For all the reasons mentioned above, dispersive methods have become a crucial tool in modern model independent analyses. Let us recall their derivation for meson-meson scattering, whose amplitude $T(s, t, u)$ depends just on two variables, since $s+t+u=\sum_{i} m_{i}^{2}$. Dispersion relations are nothing but the implementation of the analyticity of these amplitudes in the complex $s, t$ plane, which is a consequence of causality. There are different ways of building up a set of dispersion relations. The simplest one, obtained by fixing $t$, yields two main singularitites, a left hand cut, created by crossed channel interactions, and a right hand cut coming from unitarity in the physical region. One thus implements the Cauchy theorem, which in the case of a non-subtracted dispersion relation reads

$$
T(s, t)=\frac{1}{\pi} \int_{s_{t h}}^{\infty} d s^{\prime} \frac{\operatorname{Im} T\left(s^{\prime}, t\right)}{s^{\prime}-s}+\frac{1}{\pi} \int_{u_{t h}}^{-\infty} d s^{\prime} \frac{\operatorname{Im} T\left(s^{\prime}, t\right)}{s^{\prime}-s} .
$$

More subtractions may be needed depending on the behavior of high-energy Regge exchanges, but the analytic structure remains the same.

A complete study of partial-wave dispersion relations requires the expansion of all the amplitudes into partial waves, then a projection of the dispersion relations, and the final solution of both channels ( $\pi K$ and $\pi \pi \rightarrow K \bar{K}$ ) at the same time. If precision is not the main goal, there are many other simpler ways of implementing dispersion relations: one could for example unitarize Chiral Perturbation Theory in a dispersive way, which has proved to be a feasible implementation of low energy non-perturbative QCD [7, 8, 9]. There are also some works where the unphysical cuts have been approximated, but the unitarity cut is described from the data using dispersion relations [10].

In the context of resonances, all these methods produce a fairly stable calculation of the $K_{0}^{*}(700)$ pole position. There is even a rigorous dispersive result $[11,12]$. However, the $K_{0}^{*}(700)$ 
still "needs confirmation" according to the Review of Particle Physics [13]. A complete analysis of both $\pi K$ and $\pi \pi \rightarrow K \bar{K}$ channels could lead to the desired, precise and robust determination of this resonance.

The simplest dispersive set is called Forward Dispersion Relations. They are obtain by setting $t=0$ for a fixed- $t$ dispersive integral. Apart from the main advantage of its simplicity, it can also be applied up to arbitrary high energies, thus constraining the partial waves in the inelastic region, or even more, producing a strong constrain for the contributions coming from the Regge exchanges. Such a set has been proved to be very useful in previous analyses $[14,15]$.

As for the implementation of partial wave dispersion relations, the best known are the projected fixed- $t$ dispersion relations $[16,17]$, called Roy-Steiner dispersion relations. Which, in the particular case of $\pi K$ scattering read

$$
f_{l}^{I}(s)=\frac{m_{+} a_{0}^{+}}{2}+\frac{1}{\pi} \sum_{\ell} \int_{m_{+}^{2}}^{\infty} d s^{\prime} L_{l, \ell}^{I}\left(s, s^{\prime}\right) \operatorname{Im} f_{\ell}^{I}\left(s^{\prime}\right)+\frac{1}{\pi} \sum_{\ell \geq 0} \int_{4 m_{\pi}^{2}}^{\infty} d t^{\prime} L_{l, 2 \ell}^{0}\left(s, t^{\prime}\right) \operatorname{Im} g_{2 \ell}^{0}\left(t^{\prime}\right), .
$$

These equations have proved to be very useful in both the analysis of $\pi \pi$ and $\pi K$ scattering [18, $11,19]$ and in the precise determination of the parameters of the $f_{0}(500)$ [20,21]. Unfortunately, partial-wave dispersion relations cannot be applied at arbitrarily high energies, they are usually restricted to roughly the elastic regions and, in some cases, fixed- $t$ dispersion relations cannot be applied far from the real axis. On top of that, once there are different particles involved, fixed- $t$ dispersion relations fail to describe the physical regions of the $t$-channel as they are not applicable at those energies. A different set of dispersion relations [22], implemented for the relation $(s-$ $a)(u-a)=b$ is customarily used in the literature, they are called hyperbolic dispersion relations because of the curves the former equation defines on the Mandelstam Plane. The latter set has been effectively implemented for different reactions ( $\pi K, \pi \pi \rightarrow K \bar{K}, \pi N \gamma \gamma \rightarrow \pi \pi$, see [23, 11, 24, 25, 26]). The parameter $a$ is commonly chosen to enlarge the region where the dispersion relations can be applied, both in the real axis and in the complex plane.

There are two different approaches towards the implementation of dispersion relations in the literature. The first one consists on solving the dispersion relations below a matching point $s_{m}$, above which the partial waves are described using the data, together with some theoretical input for the low energy constants. Thus one does not need to include data close to threshold, as the solution is a prediction of the given set of dispersion relations. The second approach is to constrain the initial fits to the data together with the dispersion relations. The result is then a fit that describes both the data and the analytical requirements. In practice, the system is solved for the lowest partial waves as they are the only ones contributing to the low energy region. The number of subtractions depends first on the behavior of the Regge exchanges, and also on the chosen approach.

\section{Dispersive analysis}

In this work, we focus on the study of both the $\pi K$ and $\pi \pi \rightarrow K \bar{K}$ channels, and on obtaining the $K_{0}^{*}(700)$ resonance, so that the most convenient set is that of the hyperbolic dispersion relations. Nonetheless, we will also make use of fixed- $t$ dispersion relations to constrain the parameterizations of the $s$-channel in the real axis even more, as they are basically dominated by the $s$-channel inputs coming from the data. On top of that, we will finally include Forward Dispersion Relations to 

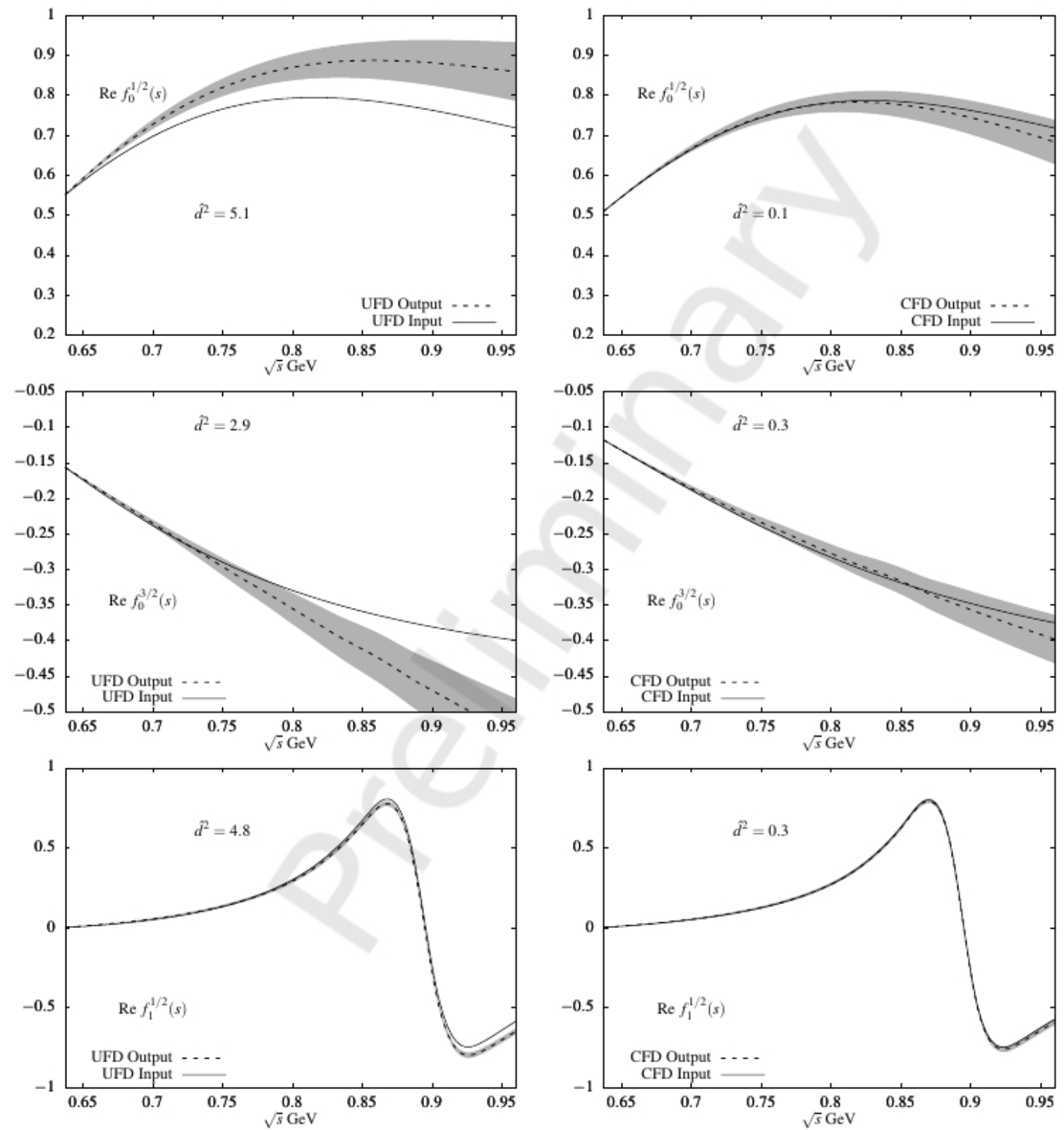

Figure 1: Preliminary checks and CFDs of the $f_{0}^{1 / 2}(s), f_{0}^{3 / 2}(s)$ and $f_{1}^{1 / 2}(s)$ partial waves.

constrain the data at higher energies, including the contributions arising from the Regge exchanges. Summarizing, we are making use of 15 dispersion relations applied to a total of 11 different partial waves.

The first stage of the work is to obtain a simple but robust description of the partial waves from the existing data sets, in order to do so we combine the implementation of several functional forms, and a consistent way of pruning the data [27]. The result is a complete description of all relevant partial waves in both channels up to $2 \mathrm{GeV}$.

Thereupon the dispersion relations are implemented and the consistency between the input (fit to the data) and the output is tested. The way to measure this deviation is through the definition of 
a distance

$$
d^{2}=\frac{1}{N} \sum_{i=1}^{N}\left(\frac{d_{i}}{\Delta d_{i}}\right)^{2},
$$

where $d_{i}$ is the difference between the "input" and "output", weighted by the uncertainty of each dispersion relation at the given energy $\sqrt{s_{i}}$. This distance is now added to the $\chi^{2}$ of every partial wave, all of them weighted by the degrees of freedom they are roughly describing. The final $d^{2}$ function has similar properties of a $\chi^{2}$. As shown in Fig. 1 there is a clear deviation between input and output. Such a behavior is due to some inputs that are not contributing appropriately to the dispersive integrals and a minimization of the whole function is hence needed. Fortunately, the $\chi^{2}$-like behavior of $d^{2}$ implies that it can be obtained by standard methods.

The last step is to constrain these data fits using the dispersion relations introduced above. As shown in Fig. 2 the differences between the unconstrained fits to data (UFD) of the scalar partial waves and the constrained ones (CFD) are small and well under the spread of the existing data. Albeit small, these deviations are crucial as all relevant partial waves are correlated through the set of dispersion relations. Once the result is found, we provide our simple, yet precise and consistent set of fits to be used in many different applications. In particular one can calculate with high accuracy interesting low energy parameters, like the scattering lengths:

$$
\begin{aligned}
& m_{\pi} a_{0}^{1 / 2}=0.22 \pm 0.01,(\text { Preliminary) } \\
& m_{\pi} a_{0}^{3 / 2}=-0.05 \pm 0.01 . \text { (Preliminary) }
\end{aligned}
$$
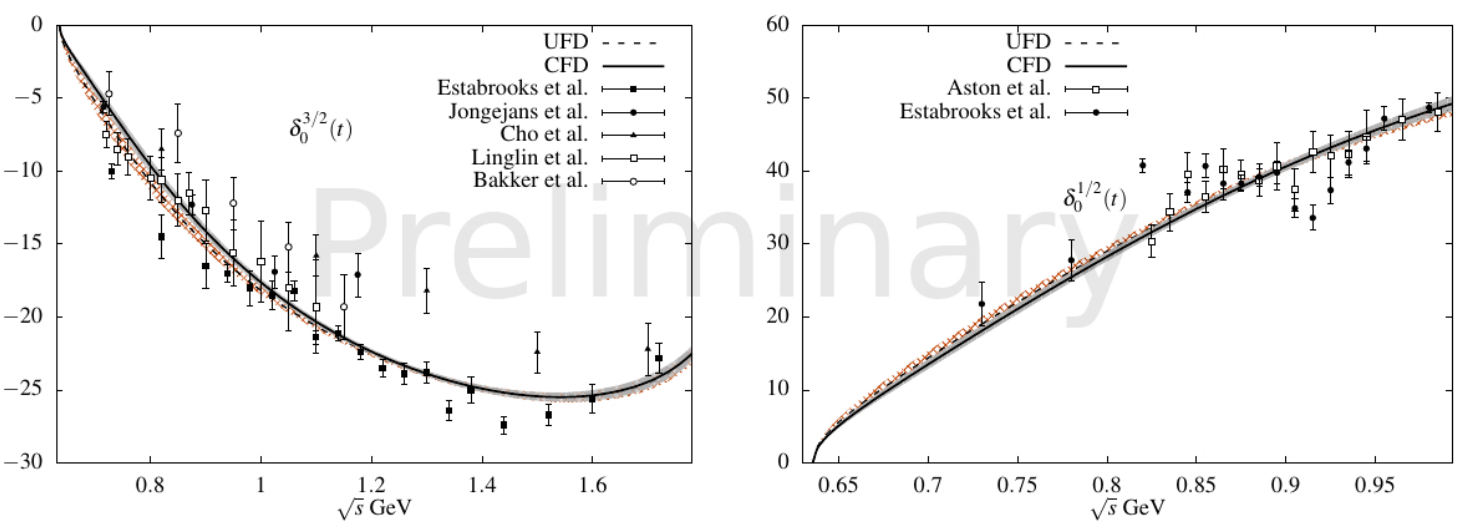

Figure 2: $f_{0}^{3 / 2}(s)$ (left) and $f_{0}^{1 / 2}(s)$ (right) preliminary CFD vs UFD partial waves.

\section{Summary}

Using the set dispersion relations explained above we have been able to constrain the data fits of both $\pi K$ and $\pi \pi \rightarrow K \bar{K}$ channels by means of a penalty $\chi^{2}$-like function. The result is the set a set of partial-wave parameterizations, which are now compatible with Roy-Steiner dispersion relations up to roughly $1 \mathrm{GeV}$, while fulfilling also Forward Dispersion Relations up to $1.6 \mathrm{GeV}$. 


\section{Acknowledgements}

JRP and AR are supported by the Spanish project FPA2016-75654-C2-2-P. AR would also like to acknowledge the financial support of the Universidad Complutense de Madrid through a predoctoral scholarship.

\section{References}

[1] A. M. Bakker et al., A determination of the $I=3 / 2 K \pi$ elastic-scattering cross section from the reaction $K^{-} n \rightarrow p K^{-} \pi^{-}$at 3 gev/c, Nucl. Phys. B24 (1970) 211.

[2] C. F. Cho and J. J. Sakurai, Tests of vector-meson dominance relations in the electric Born model for pion photoproduction, Phys.Lett. B30 (1969) 119.

[3] D. Linglin et al., $K$ - pi-elastic scattering cross-section measured in $14.3 \mathrm{gev} / \mathrm{c}$ k-p interactions, Nucl. Phys. B57 (1973) 64.

[4] P. Estabrooks, R. K. Carnegie, A. D. Martin, W. M. Dunwoodie, T. A. Lasinski and D. W. G. S. Leith, Study of $K \pi$ Scattering Using the Reactions $K^{ \pm} p \rightarrow K^{ \pm} \pi^{+} n$ and $K^{ \pm} p \rightarrow K^{ \pm} \pi^{-} \Delta^{++}$at $13 \mathrm{GeV} / \mathrm{c}$, Nucl. Phys. B133 (1978) 490.

[5] D. Aston et al., A Study of $K^{-} \pi^{+}$Scattering in the Reaction $K^{-} p \rightarrow K^{-} \pi^{+} n$ at $11 \mathrm{GeV} / c$, Nucl. Phys. B296 (1988) 493.

[6] J. R. Peláez, From controversy to precision on the $\sigma$ meson: a review on the status of the non-ordinary $f_{0}(500)$ resonance, Phys.Rept. 658 (2016) 1 [1510.00653].

[7] A. Dobado and J. R. Pelaez, The Inverse amplitude method in chiral perturbation theory, Phys. Rev. D56 (1997) 3057 [hep-ph / 9604416$].$

[8] J. A. Oller and E. Oset, N/D description of two meson amplitudes and chiral symmetry, Phys.Rev. D60 (1999) 074023 [hep-ph/ 9809337 ].

[9] J. A. Oller, E. Oset and J. R. Pelaez, Meson meson interaction in a nonperturbative chiral approach, Phys. Rev. D59 (1999) 074001 [hep-ph/ 9804209 ].

[10] H. Q. Zheng, Z. Y. Zhou, G. Y. Qin, Z. Xiao, J. J. Wang and N. Wu, The kappa resonance in s wave pi K scatterings, Nucl. Phys. A733 (2004) 235 [hep-ph/ 0310293$].$

[11] P. Buettiker, S. Descotes-Genon and B. Moussallam, A new analysis of $\pi K$ scattering from Roy and Steiner type equations, Eur. Phys. J. C33 (2004) 409 [hep-ph/ 0310283 ].

[12] S. Descotes-Genon and B. Moussallam, The $K_{0}^{*}(800)$ scalar resonance from Roy-Steiner representations of $\pi K$ scattering, Eur. Phys. J. C48 (2006) 553 [hep-ph/ 0607133 ].

[13] Particle Data Group collaboration, M. Tabanashi et al., Review of Particle Physics, Phys.Rev. D98 (2018) 030001.

[14] J. R. Pelaez and F. J. Yndurain, The Pion-pion scattering amplitude, Phys. Rev. D71 (2005) 074016 [hep-ph/0411334].

[15] J. R. Peláez and A. Rodas, Pion-kaon scattering amplitude constrained with forward dispersion relations up to 1.6 GeV, Phys. Rev. D93 (2016) 074025 [1602.084 04 ].

[16] S. M. Roy, Exact integral equation for pion pion scattering involving only physical region partial waves, Phys.Lett. 36B (1971) 353. 
[17] F. Steiner, Partial wave crossing relations for meson-baryon scattering, Fortsch. Phys. 19 (1971) 115.

[18] G. Colangelo, J. Gasser and H. Leutwyler, $\pi \pi$ scattering, Nucl. Phys. B603 (2001) 125 [hep-ph/0103088].

[19] R. García-Martín, R. Kamiński, J. R. Peláez, J. Ruiz de Elvira and F. J. Ynduráin, The Pion-pion scattering amplitude. IV: Improved analysis with once subtracted Roy-like equations up to $1100 \mathrm{MeV}$, Phys.Rev. D83 (2011) 074004 [1102.2183].

[20] I. Caprini, G. Colangelo and H. Leutwyler, Mass and width of the lowest resonance in QCD, Phys.Rev.Lett. 96 (2006) 132001 [hep-ph/ 0512364 ].

[21] R. García-Martín, R. Kaminski, J. R. Peláez and J. Ruiz de Elvira, Precise determination of the $f_{0}(600)$ and $f_{0}(980)$ pole parameters from a dispersive data analysis, Phys.Rev.Lett. 107 (2011) 072001 [1107.1635].

[22] G. E. Hite and F. Steiner, New dispersion relations and their application to partial-wave amplitudes, Nuovo Cim. A18 (1973) 237.

[23] B. Ananthanarayan and P. Buettiker, Comparison of pion kaon scattering in SU(3) chiral perturbation theory and dispersion relations, Eur. Phys. J. C19 (2001) 517 [hep-ph/ 0012023 ].

[24] M. Hoferichter, J. Ruiz de Elvira, B. Kubis and U.-G. Meißner, Roy-Steiner-equation analysis of pion-nucleon scattering, Phys. Rept. 625 (2016) 1 [1510.06039].

[25] M. Hoferichter, D. R. Phillips and C. Schat, Roy-Steiner equations for gamma gamma -> pi pi, Eur. Phys. J. C71 (2011) 1743 [1106.4147].

[26] J. R. Pelaez and A. Rodas, $\pi \pi \rightarrow K \bar{K}$ scattering up to 1.47 GeV with hyperbolic dispersion relations, Eur. Phys. J. C78 (2018) 897 [1807. 04543 ].

[27] R. Navarro Pérez, E. Ruiz Arriola and J. Ruiz de Elvira, Self-consistent statistical error analysis of $\pi \pi$ scattering, Phys.Rev. D91 (2015) 074014 [1502.03361]. 